\title{
Flashy flagella: flagellin modification is relatively common and highly versatile among the Enterobacteriaceae
}

\author{
Pieter De Maayer ${ }^{1,2^{*}}$ and Don A. Cowan ${ }^{1}$
}

\begin{abstract}
Background: Post-translational glycosylation of the flagellin protein is relatively common among Gram-negative bacteria, and has been linked to several phenotypes, including flagellar biosynthesis and motility, biofilm formation, host immune evasion and manipulation and virulence. However to date, despite extensive physiological and genetic characterization, it has never been reported for the peritrichously flagellate Enterobacteriaceae.

Results: Using comparative genomic approaches we analyzed 2,000 representative genomes of Enterobacteriaceae, and show that flagellin glycosylation islands are relatively common and extremely versatile among members of this family. Differences in the $G+C$ content of the FGls and the rest of the genome and the presence of mobile genetic elements provide evidence of horizontal gene transfer occurring within the FGl loci. These loci therefore encode highly variable flagellin glycan structures, with distinct sugar backbones, heavily substituted with formyl, methyl, acetyl, lipoyl and amino groups. Additionally, an N-lysine methylase, FliB, previously identified only in the enterobacterial pathogen Salmonella enterica, is relatively common among several distinct taxa within the family. These flagellin methylase island loci (FMIs), in contrast to the FGl loci, appear to be stably maintained within these diverse lineages.
\end{abstract}

Conclusions: The prevalence and versatility of flagellin modification loci, both glycosylation and methylation loci, suggests they play important biological roles among the Enterobacteriaceae.

Keywords: Enterobacteriaceae, Flagellum, Flagellin glycosylation, Methylation, N-lysine methylase

\section{Background}

Members of the family Enterobacteriaceae are globally distributed and include some of the most important plant, animal and clinical pathogens [1]. As a result, this family is subject to a strong sequencing bias, representing $\sim 16 \%$ of all the currently sequenced bacterial genomes [2]. The family also includes the model organism Escherichia coli $\mathrm{K}-12$ and the model for bacterial pathogenesis Salmonella enterica serovar Typhimurium [3, 4]. As these organisms can be easily manipulated and studied using currently available molecular genetic tools, they have been the basis of invaluable contributions to our current understanding of bacterial physiology, genetics and biology, including metabolism, replication, cell

\footnotetext{
* Correspondence: Pieter.demaayer@up.ac.za

${ }^{1}$ Centre for Microbial Ecology and Genomics, University of Pretoria, 0002 Pretoria, South Africa

${ }^{2}$ Department of Microbiology, University of Pretoria, 0002 Pretoria, South Africa
}

() 2016 De Maayer and Cowan. Open Access This article is distributed under the terms of the Creative Commons Attribution 4.0 International License (http://creativecommons.org/licenses/by/4.0/), which permits unrestricted use, distribution, and reproduction in any medium, provided you give appropriate credit to the original author(s) and the source, provide a link to the Creative Commons license, and indicate if changes were made. The Creative Commons Public Domain Dedication waiver (http://creativecommons.org/publicdomain/zero/1.0/) applies to the data made available in this article, unless otherwise stated. swimming motility $[3,4]$. Swimming motility in the Enterobacteriaceae is by means of peritrichous flagella; filamentous structures extruding from the surface of the bacterial cell $[5,6]$. These flagella are comprised of four main components; a motor, basal body, hook and filament. The latter is composed of up to 20,000 copies of a single protein, flagellin (FliC) [6]. The genetic basis of flagellum biosynthesis has been determined in both $E$. coli and S. enterica and involves $\sim 50$ genes, which are clustered in three distinct chromosomal regions. Gene cluster III incorporates the fliC gene coding for flagellin, as well as the flagellum capping protein $(f l i D)$ and the flagellum-specific sigma factor (fliA) [7].

As flagellar motility facilitates the rapid movement of bacterial cells, it is pivotal in allowing bacteria to move towards nutrient-rich environments and away from detrimental niches $[7,8]$. In pathogenic microorganisms, flagellar motility plays a role in colonisation, adhesion 
and biofilm formation, and as such the flagellum is considered as a virulence factor in both plant and animal pathogens [5]. However, the surface domains of the flagellin protein are highly immunogenic, and are recognized by host receptors, triggering innate and adaptive immune responses, both locally and systemically $[5,9,10]$. Motile bacterial pathogens have evolved several means to counteract flagellin recognition. For example, S. enterica and some strains of $E$. coli can alternatively express two antigenically distinct flagellin genes in a process known as phase variation $[11,12]$. Other pathogens attach sugar chains to the flagellin protein, resulting in a flagellin with distinct antigenic properties, in a process termed posttranslational flagellin glycosylation [13]. This phenomenon has been observed in a number of Gram-negative bacteria, including the human and animal pathogens Campylobacter, Aeromonas and Pseudomonas aeruginosa, as well as the phytopathogen Pseudomonas syringae [13-16]. The genes responsible for flagellin glycosylation usually occur adjacent to the flagellin gene(s) and often include sugar biosynthetic genes as well as glycosyltransferases [17]. The flagellin glycosylation loci are, however, highly variable, even among strains of the same species, resulting in highly distinct glycan structures. As such, a number of additional functions have been ascribed to flagellin glycosylation, particularly in pathogenic microorganisms, including surface recognition, adhesion, biofilm formation, mimicry of host cell surface glycans and virulence $[13,17,18]$.

To date, flagellin glycosylation has not been described in the peritrichously flagellated Enterobacteriaceae, despite the extensive body of research on this family. However, a methylase FliB has been described in S. enterica, which is involved in the posttranslational modification of the flagellin protein in this bacterium, with a predicted role in swarming motility and/or virulence $[19,20]$. Here, by means of comparative genomic approaches, we have demonstrated that flagellin glycosylation loci are relatively common among the Enterobacteriaceae. The extensive variability of these loci suggests that they code for highly variable glycans. Furthermore, we show that FliB orthologs are encoded on the genomes of a number of other enterobacterial taxa. Flagellin ornamentation with glycans and methyl groups therefore appears to be relatively common and highly variable among the Enterobacteriaceae and we postulate the possible functions associated with this characteristic.

\section{Results and discussion}

\section{Flagellin ornamentation loci are widespread among the Enterobacteriaceae}

The complete and draft genomes of 2,000 strains belonging to the family Enterobacteriaceae, encompassing 50 distinct genera, were screened for the presence of inserts within their flagellin (fliDC/AZ) loci (Additional file 1: Table S1). Inserts were located between the $\mathrm{fliC}$ and $\mathrm{fliA}$ genes in 631 of the strains. Of these, 307 (15.4\%) contained hallmarks of flagellin glycosylation islands (FGIs), including genes coding for glycosyltransferases and sugar biosynthetic enzymes (Additional file 1: Table S2). A further 302 (15.1\%) strains contained methyltransferases but no other FGI loci genes, and these inserts were termed Flagellin Methylation Islands (FMI) (Additional file 1: Table S3). The majority of the FMI loci (296 strains) encode orthologs of the $N$-lysine methylase FliB, which was first observed in Salmonella over 50 years ago [21, 22]. A distinct S-adenosylmethioninedependent methyltransferase $(\operatorname{smt} A)$ is inserted in the fliDC/AZ loci of six Pectobacterium wasabiae strains (FMI2). It should be noted that a substantial number of the strains (239 strains) included in the genomic screening of the family lack the fliDCAZ loci and are not capable of motility by means of peritrichous flagella, including Buchnera spp., Raoultella spp., Wigglesworthia spp., Phaseolibacter flectens, Serratia symbiotica and the majority of Klebsiella spp.. Thus, when considering only the peritrichously flagellate Enterobacteriaceae, 17.4 and $17.1 \%$ of the analyzed strains can be considered to contain FGI and FMI loci, respectively. A neighbour-joining phylogeny was constructed for the 2,000 Enterobacteriaceae on the basis of the concatenated amino acid sequences for the house-keeping markers GyrB, InfB and RpoB. This phylogeny (Fig. 1) shows that flagellin ornamentation loci occur in eleven of the eighteen distinct deep-branching clades, with $\mathrm{FGI}^{+}$and $\mathrm{FMI}^{+}$strains occurring in nine and seven of the clades, respectively. The majority of these clades incorporate both $\mathrm{FGI}^{+}$and $\mathrm{FMI}^{-}$strains, while $\mathrm{FGI}^{+}$strains are found exclusively in the deep-branching clades G, H, I and R, and deep-branching clades $\mathrm{B}, \mathrm{D}$ and $\mathrm{O}$ are occupied by $\mathrm{FMI}^{+}$ strains only (Fig. 1). At the genus level, FGIs are found in twenty of the fifty sampled genera, while nine genera contain FMI loci (Table 1). Three genera, Ewingella (1 strain), Franconibacter (8 strains) and Salmonella (103 strains) are exclusively comprised of $\mathrm{FMI}^{+}$strains, while eleven genera incorporate only $\mathrm{FGI}^{+}$strains. For three further genera, Brenneria (2 strains), Leclercia (2 strains) and Tatumella (4 strains), only a single strains was $\mathrm{FMI}^{+}$(Table 1$)$. Seven genera incorporated both $\mathrm{FGI}^{+}$and $\mathrm{FMI}^{+}$strains. In general, the presence of either type of flagellin ornamentation locus appeared mutually exclusive, with the exception of two strains, Pantoea agglomerans GB1 and Pantoea vagans C9-1, where the flagellin ornamentation loci contained both a $f l i B$ gene, as well as a gene coding for a GT25 family glycosyltransferase which may be involved in flagellin glycosylation.

Enterobacterial FGIs contain hallmarks of horizontal gene transfer, while the FMls are stably maintained in distinct enterobacterial lineages

The enterobacterial flagellin glycosylation islands range in size from $\sim 2.4 \mathrm{~kb}$ (Pantoea eucalypti 299R) to $\sim 23 \mathrm{~kb}$ 


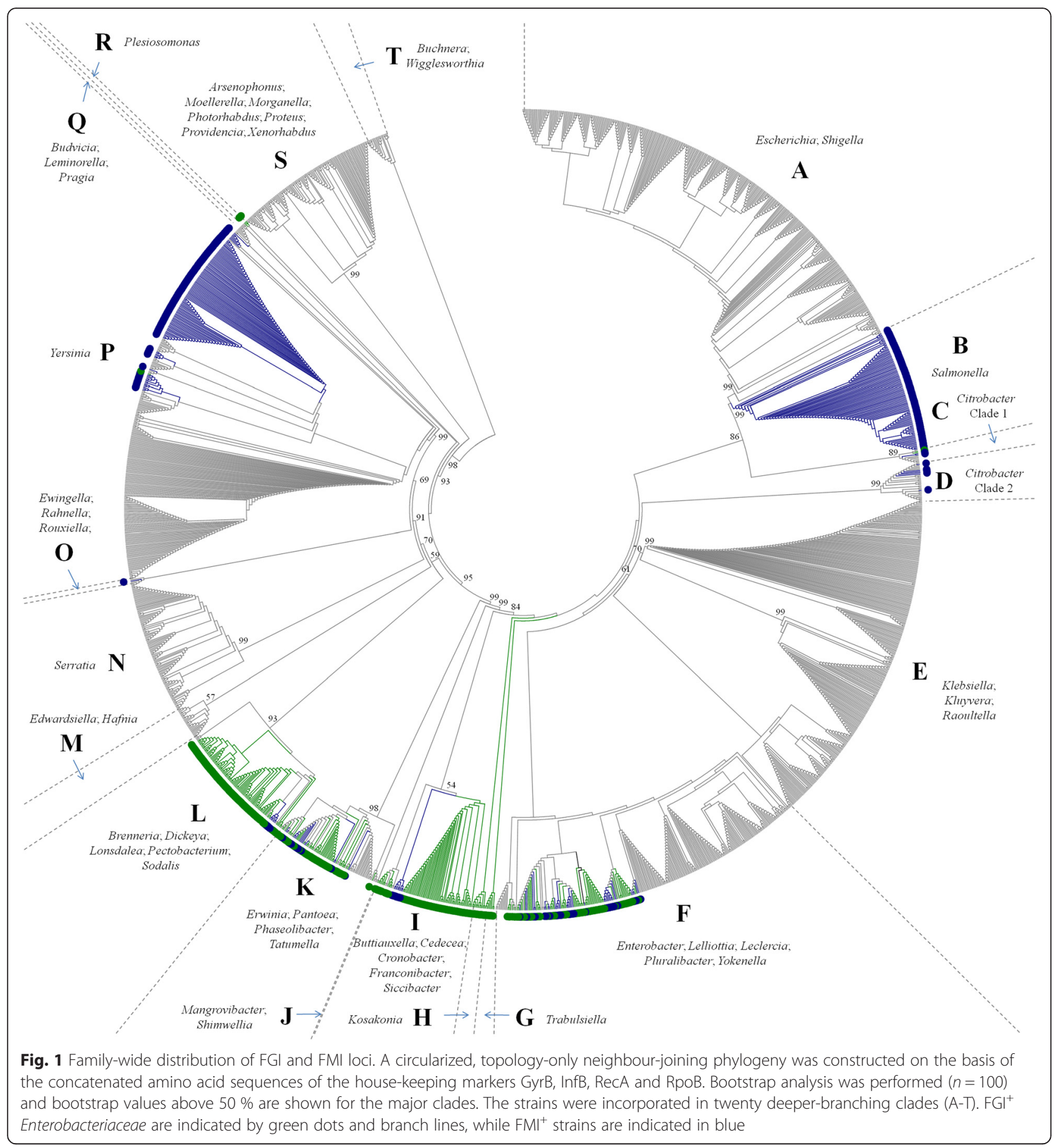

(Pantoea ananatis B1-9) and encode between one and eighteen proteins. On average the FGIs have a $\mathrm{G}+\mathrm{C}$ content $5.2 \%$ (range -17.7 to $+4.0 \%$ ) below the genomic average, suggestive of recent horizontal acquisition (Additional file 1: Table S2). Further evidence for HGT is provided by the presence of twenty-one transposase genes, with between zero and six (Kosakonia sp. CAV1151) distinct transposases encoded within the FGIs. Two distinct HNH family endonucleases (edn1 - Pantoea and Erwinia spp.; edn2 Pectobacterium carotovorum BC S2) were also identified (Additional file 1: Table S4). These homing endonucleases have been identified in bacteriophages, bacteria, archaea and eukarya and drive their own integration and replication in a host genome [23]. Several $\mathrm{FGI}^{-} / \mathrm{FMI}^{-}$enterobacteria contain inserts within the fliDC/AZ locus, with distinct putative functions not related to flagellin ornamentation 
Table 1 Prevalence and proportions of FGls and FMls for each of the sampled genera of the Enterobacteriaceae

\begin{tabular}{|c|c|c|c|c|c|c|}
\hline Genus & Clade & Strains & Species & $\mathrm{FGl}$ & FGl types & FMI \\
\hline Arsenophonus & $\mathrm{S}$ & 3 & 1 & $0(0 \%)$ & - & $0(0 \%)$ \\
\hline Brenneria & L & 2 & 2 & $1(50 \%)$ & 1 & $0(0 \%)$ \\
\hline Buchnera & $\mathrm{T}$ & 15 & 1 & $0(0 \%)$ & - & $0(0 \%)$ \\
\hline Budvicia & Q & 1 & 1 & $0(0 \%)$ & - & $0(0 \%)$ \\
\hline Buttiauxella & । & 2 & 1 & $0(0 \%)$ & - & $0(0 \%)$ \\
\hline Cedecea & । & 6 & 3 & 6 (100 \%) & 2 & $0(0 \%)$ \\
\hline Citrobacter & $C / D$ & 43 & 10 & $2(5 \%)$ & 1 & 8 (19 \%) \\
\hline Cronobacter & । & 60 & 6 & 60 (100\%) & 3 & $0(0 \%)$ \\
\hline Dickeya & L & 44 & 9 & 44 (100 \%) & 4 & $0(0 \%)$ \\
\hline Edwardsiella & M & 20 & 5 & $0(0 \%)$ & - & $0(0 \%)$ \\
\hline Enterobacter & $\mathrm{F}$ & 252 & 14 & $66(26 \%)$ & 10 & 35 (14 \%) \\
\hline Erwinia & K & 32 & 11 & $8(25 \%)$ & 4 & 1 (3 \%) \\
\hline Escherichia & A & 136 & 7 & $0(0 \%)$ & - & $0(0 \%)$ \\
\hline Ewingella & $\mathrm{O}$ & 1 & 1 & $0(0 \%)$ & - & 1 (100 \%) \\
\hline Franconibacter & । & 8 & 2 & $0(0 \%)$ & - & $8(100 \%)$ \\
\hline Hafnia & M & 8 & 2 & $0(0 \%)$ & - & $0(0 \%)$ \\
\hline Klebsiella & $\mathrm{E}$ & 279 & 5 & $0(0 \%)$ & - & $0(0 \%)$ \\
\hline Kluyvera & $E$ & 2 & 2 & $0(0 \%)$ & - & $0(0 \%)$ \\
\hline Kosakonia & $\mathrm{H}$ & 11 & 8 & 11 (100\%) & 5 & $0(0 \%)$ \\
\hline Leclercia & $\mathrm{F}$ & 2 & 1 & $1(50 \%)$ & 1 & $0(0 \%)$ \\
\hline Lelliottia & $\mathrm{F}$ & 1 & 1 & 1 (100 \%) & 1 & $0(0 \%)$ \\
\hline Leminorella & Q & 1 & 1 & $0(0 \%)$ & - & $0(0 \%)$ \\
\hline Lonsdalea & L & 4 & 2 & 4 (100 \%) & 2 & $0(0 \%)$ \\
\hline Mangrovibacter & J & 1 & 1 & 1 (100 \%) & 1 & $0(0 \%)$ \\
\hline Moellerella & $S$ & 1 & 1 & $0(0 \%)$ & - & $0(0 \%)$ \\
\hline Morganella & $S$ & 14 & 1 & $0(0 \%)$ & - & $0(0 \%)$ \\
\hline Pantoea & K & 56 & 16 & 37 (66 \%) & 8 & 12 (21 \%) \\
\hline Pectobacterium & $L$ & 52 & 5 & 45 (87 \%) & 11 & $6(12 \%)$ \\
\hline Phaseolibacter & K & 1 & 1 & $0(0 \%)$ & - & $0(0 \%)$ \\
\hline Photorhabdus & $\mathrm{S}$ & 14 & 4 & $0(0 \%)$ & - & $0(0 \%)$ \\
\hline Plesiomonas & $\mathrm{R}$ & 3 & 1 & $3(100 \%)$ & 3 & $0(0 \%)$ \\
\hline Pluralibacter & $\mathrm{F}$ & 10 & 1 & $0(0 \%)$ & - & $0(0 \%)$ \\
\hline Pragia & Q & 1 & 1 & $0(0 \%)$ & - & $0(0 \%)$ \\
\hline Proteus & S & 43 & 4 & $0(0 \%)$ & - & $0(0 \%)$ \\
\hline Providencia & $S$ & 22 & 6 & $0(0 \%)$ & - & 0 (0 \%) \\
\hline Rahnella & 0 & 4 & 1 & $0(0 \%)$ & - & 0 (0 \%) \\
\hline Raoultella & $E$ & 11 & 2 & $0(0 \%)$ & - & $0(0 \%)$ \\
\hline Rouxiella & $\mathrm{O}$ & 1 & 1 & $0(0 \%)$ & - & $0(0 \%)$ \\
\hline Salmonella & B & 103 & 2 & $0(0 \%)$ & - & $103(100 \%)$ \\
\hline Serratia & N & 111 & 12 & $0(0 \%)$ & - & $0(0 \%)$ \\
\hline Shigella & A & 243 & 4 & $0(0 \%)$ & - & $0(0 \%)$ \\
\hline Shimwellia & J & 1 & 1 & $0(0 \%)$ & - & $0(0 \%)$ \\
\hline Siccibacter & I & 4 & 2 & 4 (100 \%) & 1 & 0 (0 \%) \\
\hline Sodalis & L & 3 & 3 & $0(0 \%)$ & - & $0(0 \%)$ \\
\hline
\end{tabular}


Table 1 Prevalence and proportions of FGls and FMls for each of the sampled genera of the Enterobacteriaceae (Continued)

\begin{tabular}{|c|c|c|c|c|c|c|}
\hline Tatumella & K & 4 & 3 & $1(25 \%)$ & 1 & $0(0 \%)$ \\
\hline Trabulsiella & G & 8 & 2 & $8(100 \%)$ & 1 & 0 (0 \%) \\
\hline Wigglesworthia & $\mathrm{T}$ & 4 & 2 & $0(0 \%)$ & - & $0(0 \%)$ \\
\hline Xenorhabdus & $\mathrm{S}$ & 25 & 8 & $0(0 \%)$ & - & $0(0 \%)$ \\
\hline Yersinia & $P$ & 324 & 19 & $1(0.3 \%)$ & 1 & $128(40 \%)$ \\
\hline Yokenella & $F$ & 3 & 2 & $3(100 \%)$ & 2 & $0(0 \%)$ \\
\hline
\end{tabular}

The clade in the family-wide phylogeny (Fig. 1) in which they occur is indicated, as well as the number of different FGI types for each FGI ${ }^{+}$genus

The number of strains and \% of the total strains analysed for each genus containing FGls and FMls are indicated in bold

(Additional file 2: Figure S1; Additional file 1: Table S5). These code for proteins involved in amino acid and phosphate sugar transport and metabolism, fimbrial biogenesis and transposition. Furthermore, distinct prophages are incorporated in this region in some $\mathrm{FGI}^{-} / \mathrm{FMI}^{-}$enterobacteria. The flagellin locus of the $\mathrm{FGI}^{+}$strains Erwinia tracheiphila BuffGH and PSU-1 also incorporate an Escherichia phage D108-like prophage upstream of the FGI loci. In the former strain, this prophage is also integrated in two further locations on the chromosome, suggesting that the FGI and prophage incorporation in the flagellin locus result from two distinct integration events. A Enterobacteria P88like prophage is also incorporated in the flagellin locus of the $\mathrm{FMI}^{+}$strain Citrobacter rodentium ICC168. What role, if any, these prophages may have in flagellin ornamentation or functioning remains to be determined. The presence of a large number of highly diverse FGI and FMI loci and inserts of other functions within the fliDC/AZ loci of the Enterobacteriaceae, as well as the presence of mobile genetic elements such as transposases, endonucleases and prophages (with predicted roles in HGT), suggests that the region downstream of the $\mathrm{fliC}$ gene serves as a hot-spot for the integration of foreign DNA. However, no standard signature for integration, such as repetitive elements or tRNA genes, could be observed within the loci.

As observed for the FGIs, the FMIs, which range in size from 866 to 3,358 nucleotides in size, have an average $\mathrm{G}+\mathrm{C}$ content $7.2 \%$ (range -12.9 to -1.6 ) below the genomic average, suggesting that they have also been derived from recent HGT events (Additional file 1: Table S3). It should, however, be noted that the fliB gene is universal in several monophylogenetic lineages, including those of Salmonella enterica (100 strains) and Yersinia enterocolitica (100 strains) (Fig. 1). This would indicate that, at least in these species, the FMI is ancestral and vertically maintained throughout the species. A neighbour-joining phylogeny of the $\mathrm{FliB} / \mathrm{SmtA}$ protein sequences of all $\mathrm{FMI}^{+}$Enterobacteriaceae (Fig. 2) shows good congruence with the phylogeny on the basis of the concatenated house-keeping markers GyrB, InfB and RpoB. Pectobacterium wasabiae, which encodes a different methyltransferase (SmtA) from the other $\mathrm{FMI}^{+}$taxa (FliB), forms a distinct clade. Thus, in contrast to the
FGIs, the FMIs appear to be a more ancient trait, which is stably maintained within subpopulations of the family. In addition to the $f l i B$ gene, the FMI loci of the S. enterica serovar Typhimurium strains incorporate an insertion element, IS200, which has been used as a genetic marker for the differentiation of this serovar [21, 24]. The presence of this IS, however, does not affect the clustering of the serovar Typhimurium strains with the other $S$. enterica serovars, suggesting a distinct origin of the $f l i B$ and IS200 genetic elements. Similarly, the $f l i B$ gene in twelve Pantoea and one Erwinia strain are flanked by the $\mathrm{HNH}$ endonuclease gene $e d n 1$, which is likewise found in the $\mathrm{FGI}^{+}$Pantoea and Erwinia strains, suggesting $f l i B$ and $e d n 1$ are likely derived through two distinct HGT events.

\section{Enterobacterial flagellin glycosylation islands are highly versatile and are poorly conserved among and within species}

The protein sequence sets encoded within the FGIs were compared and orthologs were identified, where orthologs were assigned on the basis of amino acid identity and sequence coverage thresholds of $>50$ and $70 \%$, respectively. A total of 218 distinct proteins were identified within the enterobacterial FGIs, with no single protein shared among all $307 \mathrm{FGI}^{+}$taxa. A FGI typing scheme was developed on the basis of the presence/ absence of protein orthologs, with the resulting distance matrix being utilised to generate a dendrogram representing all $307 \mathrm{FGI}^{+}$strain protein datasets. Using a $50 \%$ similarity threshold (i.e. $>50 \%$ of the proteins encoded in the FGI of one strain had orthologs in the FGI of a compared strain), 42 distinct types of FGIs could be distinguished (Fig. 3, Additional file 3: Figure S2). This value increased substantially when higher cutoff values were applied, where $70 \%$ similarity and $100 \%$ identity threshold values yielded 59 and 88 distinct FGI types, respectively. The number of FGI types, regardless of the cut-off values applied, was consistently higher than the number of $\mathrm{FGI}^{+}$genera, indicating that more than one type of FGI type occurs for some genera. For example, the forty-five $\mathrm{FGI}^{+}$Pectobacterium strains could be classified into eleven distinct FGI 


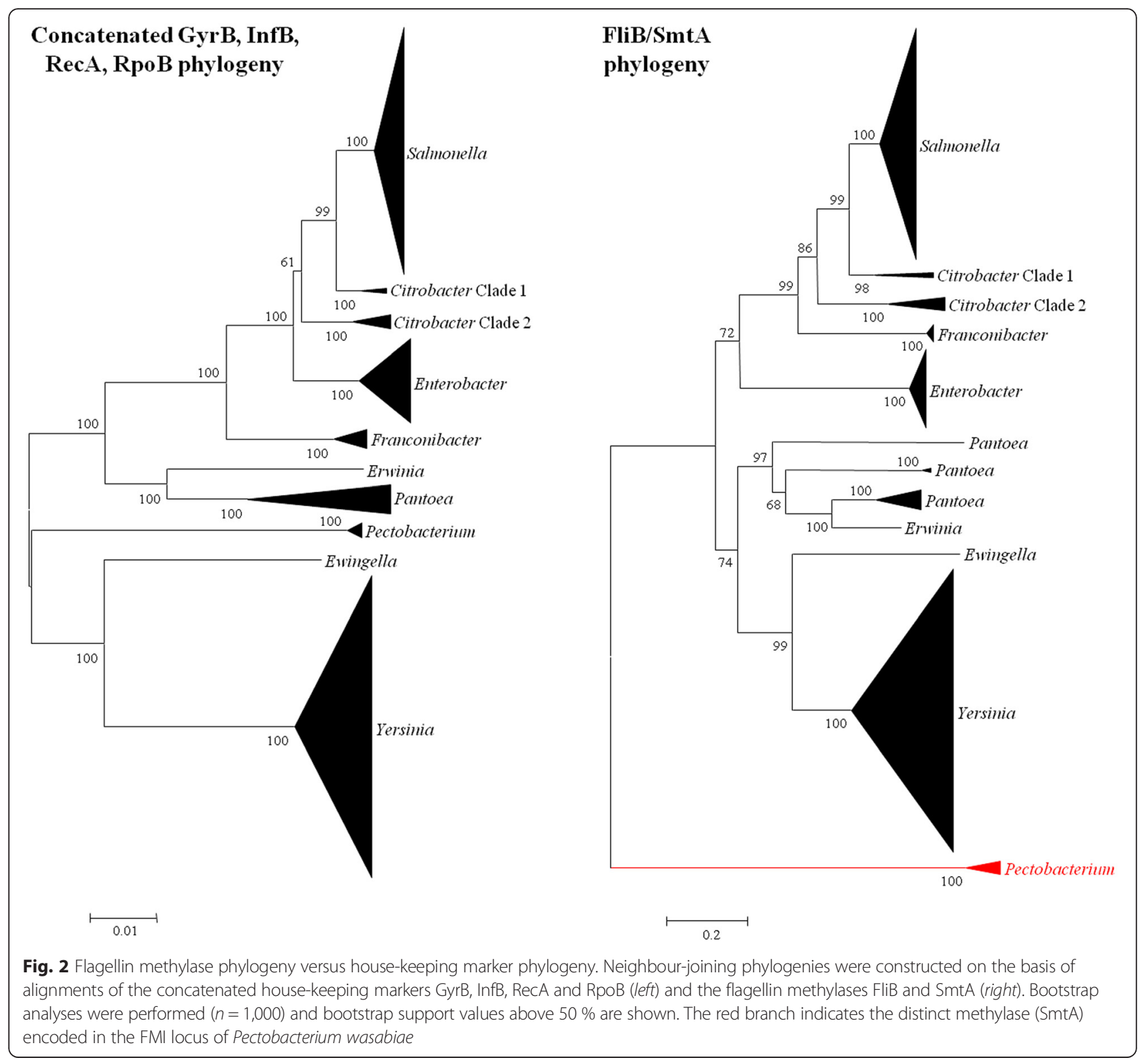

types, eight FGI types could be distinguished for the thirty-seven $\mathrm{FGI}^{+}$Pantoea spp., while three Plesiomonas shigelloides isolates had three distinct FGI types. On the other hand, monotypic FGIs could be observed for several genera, including Citrobacter, Siccibacter and Trabsuliella, with two, four and eight strains in these genera incorporated in this study (Table 1). Nevertheless, there is evidence of extensive horizontal exchange of the FGI elements. For example, FGI type 12 strains include members of the genera Dickeya, Enterobacter, Kosakonia, Lelliottia, Mangrovibacter, Pantoea, Pectobacterium and Yokenella, falling within three deeper branching clades of the house-keeping marker phylogeny (Fig. 1).
Comparison of the type 12 FGI locus of $P$. ananatis AJ13355 with that of the type 12 FGI strain Cronobacter sakazakii SP291, shows that they share a gene cluster coding for the biosynthesis of fatty acid chains which may lipoylate the glycan sugars. Similarly, a rhamnose biosynthetic cluster in the $P$. ananatis AJ13355 FGI is also found in the type 27 FGI of Pantoea stewartii M009, but not in the C. sakazakii SP291 FGI locus (Fig. 4). This suggests that the FGIs are not necessarily transferred en bloc, but instead, have developed through the incorporation of gene clusters through several distinct horizontal gene transfer events. This would further add to the structural complexity of the glycan encoded by the $\mathrm{FGI}^{+}$strains. 


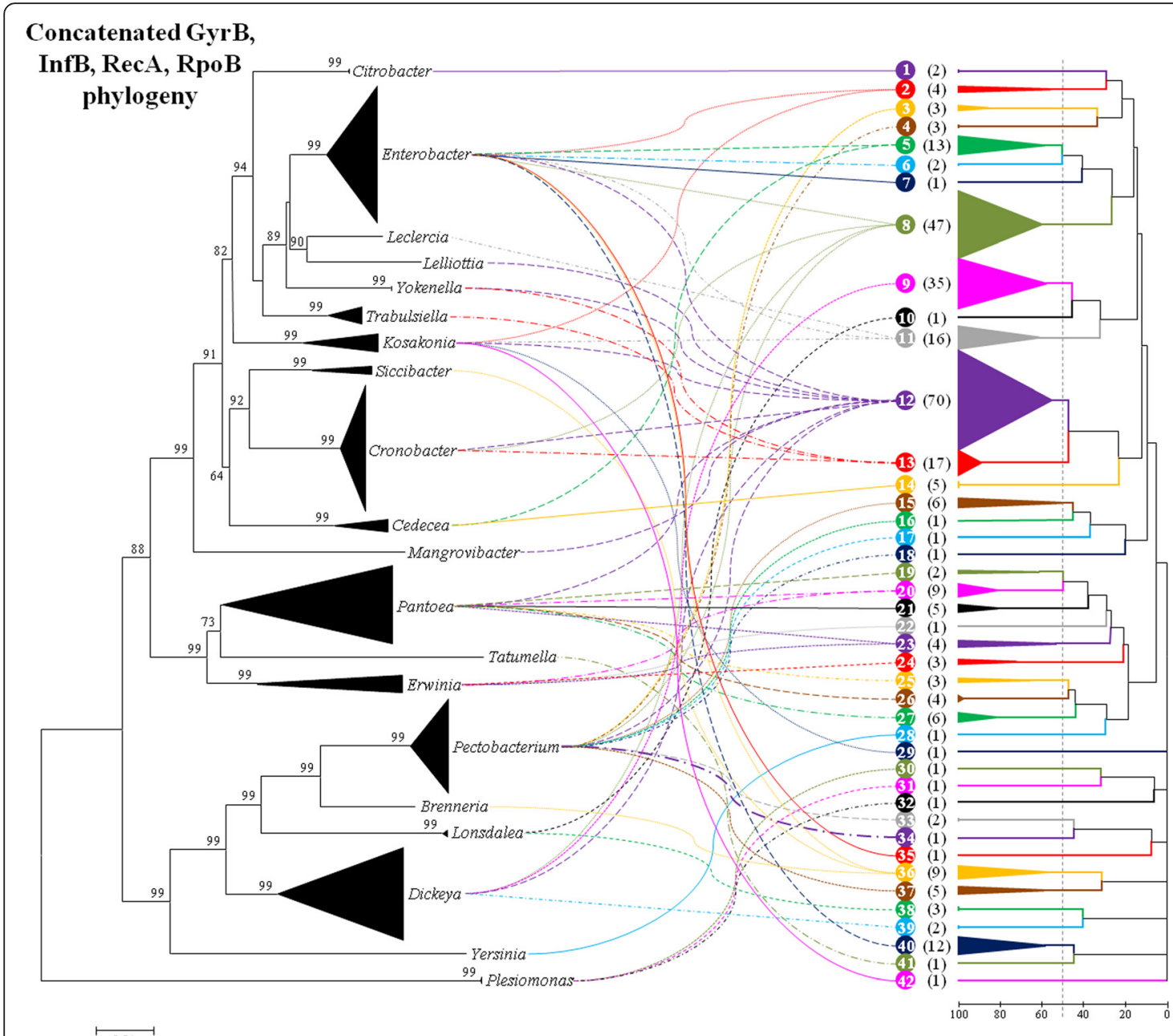

FGI typing dendrogram

Fig. 3 Flagellin glycosylation typing dendrogram. A typing dendrogram was constructed on the basis of a distance matrix representing the presence/absence of orthologs of each of the 218 distinct proteins encoded within the FGls (right). FGI types were distinguished on the basis of $50 \%$ distance cut-off values. The FGI typing dendrogram was compared against a neighbour-joining phylogeny on the basis of the amino acid sequences of the house-keeping markers GyrB, InfB, RecA and RpoB (left) for all the FGl ${ }^{+}$Enterobacteriaceaea. Bootstrap analyses were performed for the latter phylogeny and bootstrap values above $50 \%$ are shown

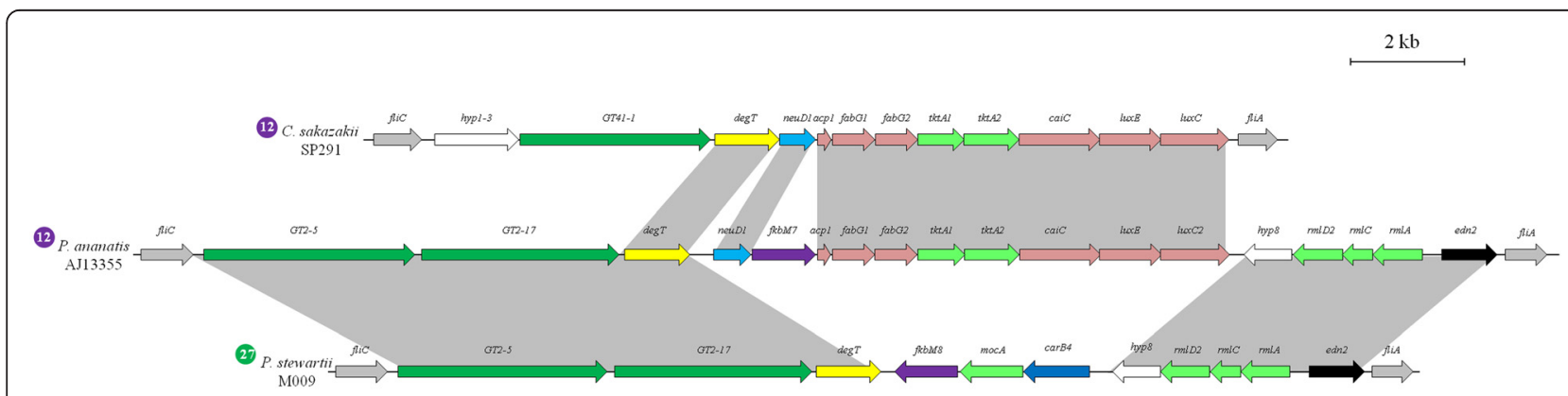

Fig. 4 Alignment of the type 12 FGl loci of P. ananatis AJ1335 and C. sakazakii SP291 and the type 27 FGl locus of P. stewartii M009. Glycosyltransferase and sugar biosynthetic genes are indicated by dark and light green arrows, respectively. Formyltransferases, methyltransferases, acetyltransferases and aminotransferases are encoded by genes represented by dark blue, purple, light blue and yellow arrows, respectively. Pink arrows indicates genes involved in fatty acid biosynthesis. Flanking genes are indicated by grey arrows, genes coding for hypothetical proteins by white arrows and black arrows indicate endonuclease (edn2) genes. The grey blocks indicate the regions of homology between the compared strains 


\section{Enterobacterial FGIs code for glycans with a wide range of backbone sugars}

Glycosyltransferases catalyse the transfer of a saccharide onto a sugar or non-sugar acceptor, and form an integral part of the post-translational protein glycosylation machinery [25]. Using the orthology cut-off values of $50 \%$ amino acid identity over $70 \%$ of the shared sequence, and BlastP analysis against the NCBI non-redundant protein database [26], forty-three distinct glycosyltransferases (GTs) could be distinguished, with between one and three GTs encoded per FGI. BlastP comparison against the Carbohydrate-Active enzymes (CAZy) database using the dbCAN pipeline $[27,28]$, classified thirtyone of these as belonging to the GT2 family, while one and eleven glycosyltransferases, respectively, were classified in the GT25 and GT41 families [25]. The presence of orthologs of GT enzymes of each family appear to be mutually exclusive, with each $\mathrm{FGI}^{+}$strains only containing GTs belonging to a single family. The GT2 enzymes transfer a broad range of saccharides, including $N$ acetyl-glucosamine, mannose, glucose, galactose, rhamnose and their derivatives [25]. Thus the type of sugars incorporated in the flagellin glycans cannot be determined on the basis of the GT2 enzyme. The GT25 family, which is found in the type 21 FGIs of five Pantoea strains, includes glucosyltransferases and galactosyltransferase and is involved in lipooligosaccharide biosynthesis in Moraxella catarrhalis, Neisseria gonorrhea and Haemophilus influenzae [29]. Similarly, the GT41 family includes peptide $N$ - $\beta$-glucosyltransferases and peptide $\beta-N$-acetylglucosaminyltransferases. The eleven distinct FGI GT41 enzymes all incorporate a conserved SPINDLY family $O$-linked $N$-acetylglucosamine transferase domain (COG3914). The presence of this domain suggests that the flagellin of $198 \mathrm{FGI}^{+}$strains, which occur in fourteen of the forty-two FGI types containing GT41 orthologs, are glycosylated with $N$-acetylglucosamine derivatives. However, considering the low level of homology among the FGI GT41 family proteins (43.6\% average amino acid identity; range 7 to $100 \%$ ) the possibility of wider transferase specificity exists.

The presence of genes involved in the biosynthesis of several distinct sugar moieties provides an insight into the flagellin glycan backbone in several $\mathrm{FGI}^{+}$enterobacteria. Most common among the sugar biosynthetic proteins are those involved in rhamnose biosynthesis, present in $28 \mathrm{FGI}^{+}$strains and six of the forty-two FGI types. The four genes required for rhamnose biosynthesis, $r m l A B C D$, are also found in the capsular polysaccharide and LPS O-antigen biosynthetic loci in a wide range of Enterobacteriaceae [30,31]. Among the $\mathrm{FGI}^{+}$ strains only one strain, Erwinia billingiae M043b (FGI type 22), encodes the full complement of Rml proteins, while all other strains lack the $r m l B$ gene coding for
dTDP-D-glucose 4,6 dehydratase, which catalyzes the second step in rhamnose biosynthesis, dehydration of dTDPD-glucose to dTDP-4-keto-6-deoxy-D-glucose [30]. Copies of $r m l B$ are, however, found elsewhere on the genome, including in the $\mathrm{O}$-antigen biosynthetic clusters, and these may complement this function in flagellin glycan biosynthesis. Other FGIs incorporate genes coding for enzymes involved in the biosynthesis of CDP-4-keto-6-deoxy-Dglucose (4 strains, FGI type 23), galactofuranose (2 strains; FGI type 39), neuraminic acid (1 strain; FGI type 16), a dTDP-6-deoxy-hex-4-ulose derivative (1 strain; FGI type 18 ), the sugar acid UDP-D-gluconate (1 strain; FGI type 36) and the pentose sugar alcohol ribitol (2 strains; FGI type 12). The diversity of sugar biosynthetic pathways incorporated in the FGIs is further highlighted by the three Plesiomonas shigelloides strains included in this study, where genes for the biosynthesis of three distinct sialic acid derivatives are present. The type 32 FGI of $P$. shigelloides 302-73 codes for ten proteins sharing $63.9 \%$ average amino acid identity with those involved in the production of legionaminic acid in the $\mathrm{O}$-antigen of Escherichia coli O161 [32], while loci for the biosynthesis of two distinct neuraminic/pseudaminic acid derivatives occur in the type 30 and 31 FGIs of P. shigelloides GN7 and ZOR011, respectively (Fig. 5).

Formylation, methylation, acetylation and lipoylation add to the complexity of the enterobacterial flagellin glycans The flagellin glycan chains of some bacterial taxa are extensively modified or substituted with methyl, formyl, acetyl or amino groups. For example, an acetamidino group is incorporated in the flagellum $\mathrm{N}$-glycan of Methanococcus maripaludis [33], while formyl and amino substitutions have been observed on sugars in the flagellin glycan of Pseudomonas aeruginosa [14]. An acetyltransferase encoded in the flagellar region of Xanthomonas oryzae is essential for flagellin glycosylation, as well as playing roles in motility, exopolysaccharide production and biofilm formation [34]. The most common modification of the flagellin glycan among the Enterobacteriaceae appears to be amination, with aminotransferases found in the FGIs of 242/307 $\mathrm{FGI}^{+}$strains. These include UDP-4-keto-6-deoxy-N-acetylglucosamine4-aminotransferases (PseC; 2 strains in two FGI types) and three distinct types of DegT/DnrJ/EryC1/StrS family aminotransferases (DegT1-2 and FdtB; 242 strains in twenty-three FGI types). Furthermore, methylation may occur in 235/307 $\mathrm{FGI}^{+}$strains. Methyltransferases belonging to three distinct families are encoded within the FGIs; S-adenosylmethionine-dependent methyltransferases (AdoMet1-14; 73 strains in fourteen FGI types), FkbM family methyltransferases (FkbM1-10; 67 strains in eight FGI types) and a demethylmenquinone family methyltransferase (Mtf; 1 strain in one FGI type). With the exception of eight GT41-11 enzymes, all remaining GT41 glycosyltransferases 


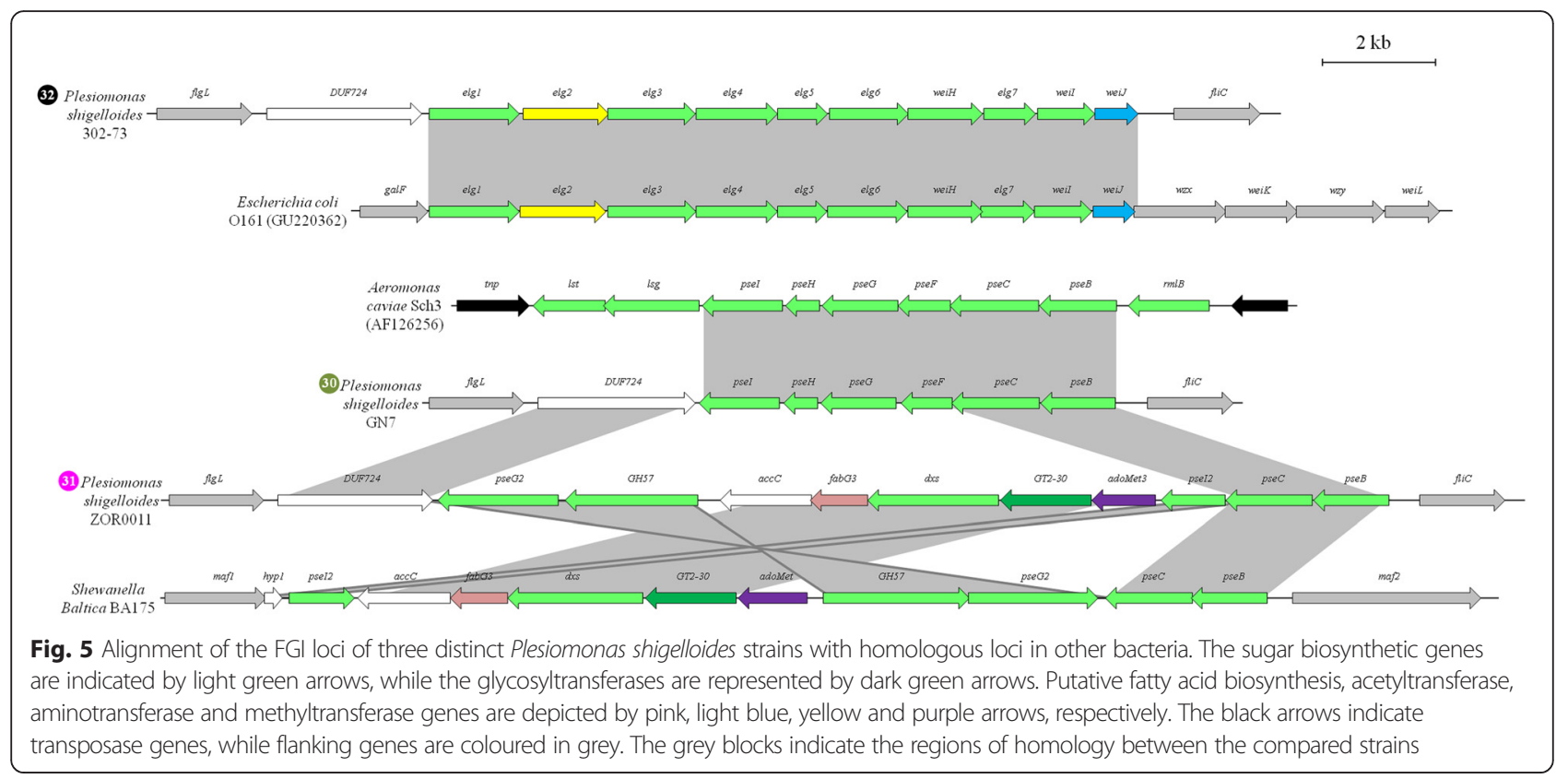

(194 strains in fifteen FGI types) as well as the GT2-30 glycosyltransferase of $P$. shiggeloides ZOR0011 also contain methyltransferase domains (AdoMet_MTases, cd02440). Two distinct types of proteins involved in $\mathrm{O}$-acetylation and two involved in $\mathrm{N}$-acetylation of a sugar moiety are encoded in the enterobacterial FGIs. These include sialic acid O-acetyltransferase (NeuD1-5; 92 strains in five FGI types), and maltose family O-acetyltransferase (WbbJ1-2; 5 strain in three FGI types), UDP-4-amino-4,6-dideoxy-Nacetyl-beta-L-altrosamine $\mathrm{N}$-acetyltransferase (PseH; 1 strain in one FGI type), and an N-acetyltransferase of unknown specificity (Nat1-5; 60 strains in two FGI types). Furthermore, formyltransferases (fmt1-4) are encoded in the FGIs of nine strains, belonging to four distinct FGI types. Some strains have identical complements of glycosyltransferase and sugar biosynthetic proteins, but differ in terms of these sugar modification enzymes. For example, the FGI type 9 strains Dickeya sp. nov 3 (S1) and sp. nov 3 (2B12) are distinguished only by the presence of a FkbM family (FkbM4) methyltransferase in the former, with an orthologs of this protein not encoded within the FGI of the latter. While the FGI of the Type 15 FGI strain Pectobacterium carotovorum CFIA1001 encodes a formyltransferase (Fmt3), P. carovotorum ICMP 5702 encodes an Oacetyltransferase (WbbJ2) within its type 15 FGI. It can thus be expected that these modification enzymes add to the versatility and complexity of the flagellin glycan structure.

The FGIs of a large number of $\mathrm{FGI}^{+}$strains also encode several proteins involved in fatty acid synthesis (FAS), including acyl carrier proteins (Acp1-2; 72 strains in three FGI types), an acyl-protein synthetase (LuxE; 73 strains in three FGI types), acyl-CoA reductases
(LuxC1-4; 80 strains in four FGI types), fatty acid CoA ligases (CaiC1-2; 71 strains plus two partial genes in two FGI types), 3-ketoacyl-(acyl-carrier protein) reductases (FabG; 71 strains in two FGI types) and betahydroxyacyl-ACP dehydratases (FabZ; one strain in one FGI type). These form part of and share orthology with proteins involved in de novo biosynthesis of fatty acid in E. coli [35]. The presence of genes coding FAS enzymes suggests that in some enterobacteria the flagellin glycan may incorporate a liposugar component.

\section{Flagellin modifications in enterobacteria: not just for looking pretty?}

A wide range of different functions have been ascribed to flagellin glycosylation. Key functions which have been reported include masking of the immunogenic epitopes on the flagellin protein, thereby avoiding host recognition during infection and evasion of the resulting host defense response, immunomodulation, surface recognition and attachment, biofilm formation, protection against proteolytic degradation as well as virulence [13, 36, 37].

In several Gram-negative pathogens, including Campylobacter and Aeromonas spp., as well as the aquatic bacterium Caulobacter crescentus, flagellin glycosylation is essential for the assembly and motility function of the flagellum [13]. The vast majority of Enterobacteriaceae, regardless of the presence of flagellin ornamentation loci are, however, described as motile. For example, FGI -/FMI ${ }^{-}$Escherichia coli strains have nevertheless been demonstrated to be motile by means of peritrichous flagella [38]. Deletion of the $f l i B$ gene also did not affect swimming motility of the $\mathrm{FMI}^{+} S$. enterica serovar 
Typhimurium LT2 [19]. It is thus likely that flagellar ornamentation is not required for flagellum synthesis, maintenance or motility and that it may serve alternative functions within the Enterobacteriaceae.

The isolation sources of the $\mathrm{FGI}^{+}$and $\mathrm{FMI}^{+}$strains were recorded (Table 2), with the majority of the $\mathrm{FGI}^{+}$enterobacteria being isolated from plant and human hosts. The vast majority of these $(85.6 \%$ of the strains from these hosts and $62.5 \%$ of the $\mathrm{FGI}^{+}$strains) were pathogens or of clinical relevance, suggesting that the FGI may play a role in pathogenesis and/or virulence. Given the extensive variability in gene content of the FGIs, even among strains of the same species, the masking of the immunogenic flagellin from detection by the host is a plausible function for the enterobacterial flagellin glycans. This has been observed in several human pathogens, including Burkholderia cenocepacia and Campylobacter spp., as well as the phytopathogen Pseudomonas syringae $[15,16,39]$. The versatile structures of the Pectobacterium carotovorum FGI, which belong to nine distinct FGI types, may aid in the infection of the same host plant by $P$. carotovorum strains with different FGI types, without host detection and associated defense responses. Alternatively, flagellin glycosylation may serve as a determinant of host specificity, as observed in $P$. syringae pv. glycinea, where the flagellin glycan can induce a hyper-sensitive response in non-host plants [40]. The FGIs are not restricted to pathogens. However, as there is an apparent bias towards the sequencing of plant and animal-pathogenic isolates, the prevalence of FGIs in environmental isolates may be underestimated. These factors, along with the extensive variability of the FGIs and the glycan structures they may encode, would suggest that alternative roles for flagellin glycosylation among the various enterobacterial taxa must be considered.

The FMIs are mostly observed in the genomes of human and animal isolates, which is probably due to the universal occurrence of this locus in S. enterica and $Y$. enterocolitica, where vertebrate hosts serve as a natural reservoir for these bacterial taxa. The genomes of only a limited number of plant-associated bacteria contain an FMI, and these include the distinct flagellin methylation island (FMI2) observed in P. wasabiae. The prevalence of the $f l i B$ among clinical S. enterica isolates, and the observation that deletion of this gene did not directly affect swimming motility, have led to the hypothesis that flagellin methylation plays a role in virulence [19]. Recently, a $S$. enterica fliB mutant was shown to be defective in swarming motility [20]. Swarming is the coordinated multi-

Table 2 Isolation sources of the $\mathrm{FGl}^{+}$and $\mathrm{FMI}^{+}$enterobacterial taxa

\begin{tabular}{|c|c|c|c|c|}
\hline Locus & Isolation Source & \# strains (\% positive strains) & Specific source/relationship with host & \#strains (\% source) \\
\hline \multirow[t]{11}{*}{$\overline{\mathrm{FGl}}$} & \multirow[t]{2}{*}{ Plant } & \multirow[t]{2}{*}{$129(42.0 \%)$} & Pathogen & $103(79.8 \%)$ \\
\hline & & & Saprophyte & $26(20.2 \%)$ \\
\hline & \multirow[t]{2}{*}{ Human } & \multirow[t]{2}{*}{$96(31.3 \%)$} & Clinical & $91(94.8 \%)$ \\
\hline & & & Commensal & $5(5.2 \%)$ \\
\hline & \multirow[t]{4}{*}{ Environmental } & \multirow[t]{4}{*}{$55(17.9 \%)$} & Fresh/Marine water & $32(58.2 \%)$ \\
\hline & & & Food & $12(21.8 \%)$ \\
\hline & & & Soil & $9(16.4 \%)$ \\
\hline & & & Other & $2(3.6 \%)$ \\
\hline & \multirow[t]{2}{*}{ Animal } & \multirow[t]{2}{*}{$12(3.9 \%)$} & Vertebrate & $1(8.3 \%$ \\
\hline & & & Invertebrate & $11(91.7 \%)$ \\
\hline & Not available & $15(4.9 \%)$ & & \\
\hline \multirow[t]{10}{*}{ FMI } & Human & $137(45.4 \%)$ & Clinical & $137(100 \%)$ \\
\hline & \multirow[t]{2}{*}{ Animal } & \multirow[t]{2}{*}{79 (26.2 \%) } & Vertebrate & 77 (97.5\%) \\
\hline & & & Invertebrate & $2(2.5 \%)$ \\
\hline & \multirow[t]{4}{*}{ Environmental } & \multirow[t]{4}{*}{$42(13.9 \%)$} & Food & $24(57.1 \%)$ \\
\hline & & & Soil & $7(16.7 \%)$ \\
\hline & & & Fresh/Marine water & $4(9.5 \%)$ \\
\hline & & & Other & $7(16.7 \%)$ \\
\hline & \multirow[t]{2}{*}{ Plant } & \multirow[t]{2}{*}{$21(7.0 \%)$} & Pathogen & 7 (33.3 \%) \\
\hline & & & Saprophyte & 14 (66.7 \%) \\
\hline & Not available & $23(7.6 \%)$ & & \\
\hline
\end{tabular}


cellular movement across a semisolid surface and this behaviour plays a role in biofilm formation, colony spread, resistance to antimicrobials, intercellular communication within a swarm, and pathogenesis [20, 41]. Whether flagellin glycosylation plays a role in this phenotype in the other $\mathrm{FMI}^{+}$enterobacteria is unclear. However, swarming motility has been observed in bacteria lacking flagellin methylation islands, such as E. coli [42], and thus it is plausible the flagellin methylation may play a role in other phenotypes.

\section{Conclusions}

Despite the huge body of research conducted on the Enterobacteriaceae, flagellin glycosylation has to date not been reported for members of this family. Here we provide genomic evidence of flagellin glycosylation islands in a relatively large number of strains, representing a substantial cross section of the taxa in this family. Furthermore, the $f l i B$ gene, coding for an $\mathrm{N}$-lysine methylase is present not only in S. enterica strains, but also several other taxa, including Yersinia, Enterobacter, Franconibacter and Pantoea spp. This suggests flagellum ornamentation is a relatively common trait among the Enterobacteriaceae, in particular among pathogenic strains. This may, however, be as a result of a focus towards genome sequencing of strains of anthrophocentric relevance, and future genome sequencing endeavours may shed further light of the prevalence of flagellin ornamentation loci among environmental strains and species. The extensive genetic variability observed among the flagellin glycosylation islands, in terms of the distinct glycosyltransferases, sugar biosynthetic pathways, even among strains of the same species, suggest they are likely to encode glycans of equally diverse structure and functioning. This may be even further complexed by the variable modification, by means of lipoylation, formylation, acetylation and methylation, of the glycan backbone and/or the flagellin protein.

While genes coding for glycosyltransferases are common features among the enterobacterial FGIs, sugar biosynthetic pathways are encoded within only a limited number of taxa. Furthermore, as exemplified by the rhamnose biosynthetic pathway incorporated in the FGI of several taxa which lacks orthologs of the $r m l B$ gene, incomplete or absent pathways may affect the flagellin glycan structure. It is more likely, however, that sugar biosynthetic pathways located elsewhere on the chromosome, or forming part of other glycan biosynthetic processes, such as LPS biosynthesis, may complement this function. As a result, structural characterization of the distinct glycans should be undertaken in order to determine the exact structures of the enterobacterial flagellin glycans. The absence of flagellin ornamentation loci in many Enterobacteriaceae which have been demonstrated to possess the capacity of flagellar swimming motility suggests that this trait is not central to the swimming phenotype. Flagellin glycosylation or methylation is thus likely to serve alternative functions among the Enterobacteriaceae. Considering the extensive diversity of the FGI and FMI loci, however, we suggest that the role played by these modifications would need to be determined on a case by case basis. The data presented here should thus serve as a primer for further research into the flagellin ornamentation structures as well as the roles that these traits may play in the various enterobacterial taxa in which they occur.

\section{Methods}

Identification, annotation and characterization of the flagellin ornamentation islands

The genome sequences of 2,000 members of the family Enterobacteriaceae were obtained from the NCBI database. The genomes were selected to be representative of the fifty genera for which genome sequences are available. For those species for which more than 100 genomes are available, genome completeness was used as the criterion for subsampling. The FliC and FliA/FlgL proteins of Escherichia coli K-12 substr. MG1655 (NCBI Acc. \# AAC74990.1 and AAC74989.1) were used in a localized tBlastN analysis with BioEdit v. 7.1.11 [43] to identify the genomic locations of the coding genes in the 2,000 enterobacteria. The $f l i C$ to $f l i A / f l g L$ regions were extracted and the $\mathrm{G}+\mathrm{C}$ contents of the interior fragments were determined using BioEdit [43]. The regions were structurally annotated using Prokaryotic GeneMark.hmm v. 2 [44] and the protein sequences were functionally annotated by BlastP analysis against the NCBI non-redundant protein database and the NCBI Conserved Domain Database using CD-search $[45,46]$. Putative glycosyltransferases were classified according to the Carbohydrate Active enzymes database using the dbCAN Blast tool $[27,28]$. Orthologs among the FGI protein datasets were identified using localized BlastP analyses with BioEdit [43]. Orthology was assumed for those proteins which shared $>50 \%$ amino acid identity over $70 \%$ of the alignment length.

\section{Phylogenetic analyses}

A phylogeny was constructed for the 2,000 enterobacterial strains using the concatenated amino acid sequences of four house-keeping markers commonly used for delineation of the Enterobacteriaceae, Gyrase B (GyrB), translation initiation factor IF-2 ( $\operatorname{nfB}$ ), recombinase A (RecA) and RNA polymerase beta subunit (RpoB). Alignments were generated using the MAFFT v. 7 server and neighbour-joining trees were constructed using the Molecular Evolutionary Genetics Analysis (MEGA) v 5.0.3 software package with 
the default parameters and bootstrap analysis $(n=100$ replicates) [47, 48]. Similarly, neighbour-joining phylogenies were constructed for the $\mathrm{FGI}^{+}$and $\mathrm{FMI}^{+}$ strains, with bootstrap analyses ( $n=1,000$ replicates). The presence/absence of proteins in the FGI regions were scored, where present orthologs $=1$, while absent orthologs $=0$. The resultant matrix was used to generate a distance matrix with Bionumerics v 6.6 (Applied Maths N.V., Belgium) using the parameters: absolute values and Pearson's correlation. This distance matrix was subsequently used to generate an Unweighted Pair Group Method with Arithmetic Mean (UPGMA) dendrogram using Phylip v 3.69 and visualized with MEGA v $5.0 .3[48,49]$. A distance value cut-off value of $50 \%$ was used to discriminate between the FGI types.

\section{Additional files}

Additional file 1: Table S1. Enterobacteriaceae strains analysed in this study. The deep-branching clade to which they belong (Fig. 1), the presence/ absence of fliDCAZ loci, the NCBI accession numbers of the contigs on which these loci occur, and presence/absence of FGI and FMl loci are indicated. Table S2. Characteristics of FGI Enterobacteriaceae and their flagellin glycosylation islands. The isolation sources and deep-branching clade to which they belong in the Enterobacteriaceae phylogeny (Fig. 1) are indicated for each of the $\mathrm{FGl}^{+}$ enterobacterial strains. The sizes, $\mathrm{G}+\mathrm{C}$ contents, $\mathrm{G}+\mathrm{C}$ deviation from the rest of the genome and number of proteins encoded on each FGl are shown. Table S3. Characteristics of FMI ${ }^{+}$Enterobacteriaceae and their flagellin methylation islands. The isolation sources and deep-branching clade to which they belong in the Enterobacteriaceae phylogeny (Fig. 1) are indicated for each of the $\mathrm{FMI}^{+}$enterobacterial strains. The sizes, $\mathrm{G}+\mathrm{C}$ contents, $\mathrm{G}+\mathrm{C}$ deviation from the rest of the genome and number of proteins encoded on each FMI are shown. Table S4. Annotations of the proteins encoded on the enterobacterial FGls. The number of strains and genera in which orthologs of each distinct protein are encoded within the FGls are indicated, as well as the closest non-enterobacterial Blast hit, obtained by BlastP analysis against the NCBI non-redundant protein database. Orthologs were only considered among the top 500 BLAST hits and for those orthologs with $>30 \%$ amino acid identity to the query protein. The putative function and conserved domains observed after BLAST analyses against the NCBI protein and conserved domain databases are shown. Table S5. Genomics inserts in the fliDCAZ loci of FGl'/FMI' Enterobacteriaceae. The insert size, $\mathrm{G}+\mathrm{C}$ content, $\mathrm{G}+\mathrm{C}$ deviation from the rest of the genome, number of proteins encoded and putative functions of the encoded proteins in each insert are shown. (XLSX $234 \mathrm{~kb})$

Additional file 2: Figure S1. Schematic diagrams of inserts within the fliDCAZ loci of $\mathrm{FGI}^{-} / \mathrm{FMI}^{-}$Enterobacteriaceae. Flanking genes are indicated by yellow arrows, predicted phage genes by blue arrows, fimbrial biogenesis genes by grey arrows and sugar/amino acid transporter genes by orange arrows. Black arrows indicate predicted transposase or endonuclease genes, while the red arrows indicate genes with disrupted reading frames. The flagellin glycan biosynthetic genes in the $\mathrm{FGI}^{+}$strains E. tracheiphila Buff/ PSU-1 are indicated by green arrows, upstream of the predicted phage integration site. (TIF $471 \mathrm{~kb}$ )

Additional file 3: Figure S2. Schematic diagrams of stereotypical flagellin glycosylation islands of the forty-two distinct FGl types. Glycosyltransferase and sugar biosynthetic genes are indicated by dark and light green arrows, respectively. Formyltransferases, methyltransferases, acetyltransferases and aminotransferases are encoded by genes represented by dark blue, purple, light blue and yellow arrows, respectively. Pink arrows indicates genes involved in fatty acid biosynthesis. Flanking genes are indicated by grey arrows, genes coding for hypothetical proteins or involved in functions with no relative known function in flagellin glycosylation by white arrows and black arrows indicate transposes and endonuclease genes. (TF 3168 kb)

\section{Abbreviations}

CDS: protein coding sequence; FGl: flagellin glycosylation island; FMl: flagellin methylation island; GT: Glycosyltransferase; HGT: horizontal gene transfer.

\section{Funding}

This study was partially supported by the National Research Foundation of South Africa (NRF grant \#: 91447).

\section{Availability of data and materials}

All genome sequences incorporated in this study are publically available in the NCBI Genome database. The NCBI accession numbers for the contigs/ chromosomes on which the target loci are found are indicated in Additional file 1: Table S1.

\section{Authors' contributions}

PDM and DAC conceived the study. PDM performed data analysis, PDM and DAC wrote the original manuscript and contributed to the final version. Both authors read and approved the final manuscript.

\section{Authors' information}

PDM (PhD, Dr) is a National Research Foundation of South Africa Research Career Advancement Fellow at the Centre for Microbial Ecology and Genomics, University of Pretoria. DAC (PhD, Prof) is the Director of the Centre for Microbial Ecology and Genomics, and the Genomics Research Institute, University of Pretoria.

\section{Competing interests}

The authors declare that they have no competing interests.

\section{Consent for publication}

Not Applicable.

\section{Ethics approval and consent to participate}

Not Applicable.

Received: 30 March 2016 Accepted: 12 May 2016

Published online: 20 May 2016

\section{References}

1. Imhoff JF. Enterobacteriales". In: Brenner DJ, Krieg NR, Staley JT, Garrity GM, Boone DR, Vos P, Goodfellow M, Rainey FA, Schleifer K-H, editors. Bergey's Manual ${ }^{\oplus}$ of Systematic Bacteriology: Vol 2 The Proteobacteria Part B The Gammaproteobacteria. Boston, MA: Springer US; 2005. p. 587-850.

2. Reddy TB, Thomas AD, Stamatis D, Bertsch J, Isbandi M, Jansson J, et al. The Genomes OnLine Database (GOLD) v. 5: a metadata management system based on a four level (meta)genome project classification. Nucleic Acids Res. 2015:43:D1099-106.

3. Blount ZD. The unexhausted potential of E. Coli eLife. 2015;4:e05826.

4. Ohl ME, Miller SI. Salmonella: a model for bacterial pathogenesis. Annu Rev Med. 2001;52:259-74.

5. Josenhans $C$, Suerbaum $S$. The role of motility as a virulence factor in bacteria. Int J Med Microbiol. 2002;291:605-14.

6. Macnab RM. How bacteria assemble flagella. Annu Rev Microbiol. 2003;57: $77-100$

7. Soutourina OA, Bertin PN. Regulation cascade of flagellar expression in Gram-negative bacteria. FEMS Microbiol Rev. 2003;27:505-23.

8. Fenchel T. Microbial behavior in a heterogeneous world. Science. 2002;296: 1068-71.

9. Hayashi F, Smith KD, Ozinsky A, Hawn TR, Yi EC, Goodlett DR, et al. The innate immune response to bacterial flagellin is mediated by Toll-like receptor 5. Nature. 2001;410:1099-103.

10. Tarahomjoo S. Utilizing bacterial flagellins against infectious diseases and cancers. Antonie Van Leeuwenhoek. 2014;105:275-88.

11. Aldridge PD, Wu C, Gnerer J, Karlinsey JE, Hughes KT, Sachs MS. Regulatory protein that inhibits both synthesis and use of the target protein controls flagellar phase variation in Salmonella enterica. Proc Natl Acad Sci U S A. 2006;103:11340-5.

12. Liu B, Hu B, Zhou Z, Guo D, Guo X, Ding P, et al. A novel non-homologous recombination-mediated mechanism for Escherichia coli unilateral flagellar phase variation. Nucleic Acids Res. 2012;40:4530-8. 
13. Logan SM. Flagellar glycosylation - a new component in the motility repetoire? Microbiol. 2006;152:1249-62.

14. Miller W, Matewish MJ, McNally DJ, Ishiyama N, Anderson EM, Brewer D, et al. Flagellin glycosylation in Pseudomonas aeruginosa PAK requires the O-antigen biosynthesis enzyme WbpO. J Biol Chem. 2008;283:3507-18.

15. Szymanski CM, Logan SM, Linton D, Wren BW. Campylobacter - a tale of two protein glycosylation systems. Trends Microbiol. 2003;11:233-8.

16. Taguchi F, Suzuki T, Takeuchi K, Inagaki Y, Toyoda K, Shiraishi T, et al. Glycosylation of flagellin from Pseudmonas syringae pv. tabaci 6605 contributes to evasion of host tobacco plant surveillance systems.Physio Mol. Plant Pathol. 2009;74:11-7.

17. Power PM, Jennings MP. The genetics of glycosylation in Gram-negative bacteria. FEMS Microbiol Lett. 2003;218:211-22.

18. Merino S, Tomás JM. Gram-negative flagella glycosylation. Int J Mol Sci. 2014;15:2840-57.

19. Frye J, Karlinsey JE, Felise HR, Marzolf B, Dowidar N, McClelland M, et al. Identification of new flagellar genes of Salmonella enterica serovar Typhimurium. J Bacteriol. 2006;188:2233-43.

20. Deditius JA, Felgner S, Spöring I, Kühne C, Frahm M, Rohde M, et al. Characterization of novel factors involved in swimming and swarming motility in Salmonella enterica serovar Typhimurium. PLoS One. 2015;10: e013531.

21. Burnens AP, Stanley J, Sack R, Hunziker P, Brodard I, Nicolet J. The flagellin $\mathrm{N}$-methylase gene fliB and an adjacent serovar-specific IS200 element in Salmonella typhimurium. Microbiol. 1997;143:1539-47.

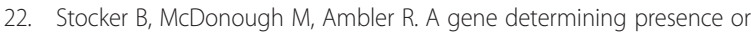
absence of $\varepsilon$-N-methyl-lysine in Salmonella flagellar protein. Nat 1961;189: 556-558.

23. Yahara K, Fukuyo M, Sasaki A, Kobayashi I. Evolutionary maintenance of selfish homing endonuclease genes in the absence of horizontal gene transfer. Proc Natl Acad Sci U S A. 2009;106:18861-6.

24. Echeita MA, Herrera S, Usera MA. Atypical, fljB-negative Salmonella enterica subsp. enterica strain of servar 4,5,12:i:- appears to be a monophasic variant of serovar Typhimurium. J Clin Microbiol. 2001;39:2981-3.

25. Coutinho PM, Deleury E, Davies GJ, Henrissat B. An evolving hierarchical family classification for glycosyltransferases. J Mol Biol. 2003;328:307-17.

26. Altschul SF, Gish W, Miller W, Myers EW, Lipman DJ. Basic local alignment search tool. J Mol Biol. 1990;215:403-10.

27. Lombard V, Golaconda Ramulu H, Drula E, Coutinho PM, Henrissat B. The carbohydrate-active enzymes database (CAZy) in 2013. Nucleic Acids Res. 2014;42:D490-5.

28. Yin Y, Mao X, Yang JC, Chen X, Mao F, Xu Y. dbCAN: a web resource for automated carbohydrate-active enzyme annotation. Nucleic Acids Res. 2012; 40:W445-51.

29. Edwards KJ, Allen S, Gibson BW, Campagnari AA. Characterization of a cluster of three glycosyltransferase enzymes essential for Moraxella catarrhalis lipooligosaccharide assembly. J Bacteriol. 2005;187:2939-47.

30. Lin $Q$, Reeves PR. Genetic variation of dTDP-L-rhamnose pathway genes in Salmonella enterica. Microbiol. 2000;146:2291-307.

31. Pan Y-J, Lin T-L, Chen C-T, Chen Y-Y, Hsieh P-F, Hsu C-R, et al. Genetic analysis of capsular polysaccharide synthesis gene clusters in 79 capsular types of Klebsiella spp. Sci Rep. 2015:5:15573.

32. Li X, Perepelov AV, Wang Q, Senchenkova SN, Liu B, Shevelev SD, et al. Structural and genetic characterization of the O-antigen of Escherichia coli O161 containing a derivative of a higher acidic diamino sugar, legionaminic acid. Carbohydr Res. 2010;345:1581-7.

33. Jones GM, Wu J, Ding Y, Uchida K, Aizawa S, Robotham A, et al. Identification of genes involved in the acetamidino group modification of the flagellin N-linked glycan of Methanococcus maripaludis. J Bacteriol. 2012; 194:2693-702.

34. Li H, Yu C, Chen H, Tian F, He C. PXO 00987, a putative acetyltransferase, is required for flagellin glycosylation, and regulates flagellar motility, exopolysaccharide production, and biofilm formation in Xanthomonas oryzae pv. oryzae. Microb Pathog. 2015;85:50-7.

35. Chan DI, Vogel HJ. Current understanding of fatty acid biosynthesis and the acyl carrier protein. Biochem J. 2010;430:1-19.

36. Nothaft H, Szymanski CM. Protein glycosylation in bacteria: sweeter than ever. Nat Rev Microbiol. 2010;8:765-78.

37. Schmidt MA, Riley LW, Benz I. Sweet new world: glycoproteins in bacterial pathogens. Trends Microbiol. 2003;11:554-61.
38. Zhou M, Yang Y, Chen P, Hu H, Hardwidge PR. More than a locomotive organelle: flagella in Escherichia coli. Appl Microbiol Biotechnol. 2015;99: 8883-90.

39. Hanuszkiewicz A, Pittock P, Humphries F, Moll H, Rosales AR, Molinaro A et al. Identification of the flagellin glycosylation system in Burkholderia cenocepacia and the contribution of glycosylated flagellin to evasion of human innate immune responses. J Biol Chem. 2014;289:19231-44.

40. Takeuchi K, Taguchi F, Inagaki Y, Toyoda K, Shiraishi T, Ichinose Y. Flagellin glycosylation island in Pseudomonas syringae pv. glycinea and its role in host specificity. J Bacteriol. 2003;185:6658-65.

41. Partridge JD, Harshey RM. Sawrming: flexible roaming plans. J Bacteriol. 2013;195:909-18.

42. Inoue T, Shingaki R, Hirose S, Waki K, Mori H, Fukui K. Genome-wide screening of genes required for swarming motility in Escherichia coli K-12. J Bacteriol. 2007:189:950-7.

43. Hall TA. BioEdit: a user-friendly biological sequence alignment editor and analysis program for Windows 95/98/NT. Nucleic Acids Symp Ser. 1999:41:95-8.

44. Besemer J, Lomsadze A, Borodovsky M. GeneMarkS: a self-training method for prediction of gene starts in microbial genomes. Implications for funding sequence motifs in regulatory regions. Nucleic Acids Res. 2001;29:2607-18.

45. Marchler-Bauer A, Bryant SH. CD-Search: protein domain annotations on the fly. Nucleic Acids Res. 2004;32:W327-31.

46. Marchler-Bauer A, Derbyshire MK, Gonzales NR, Lu S, Chitsaz F, Geer LY, et al. CDD: NCBI's conserved domain database. Nucleic Acids Res. 2015:43:D222-6.

47. Katoh K, Standley DM. MAFFT multiple sequence alignment version 7: improvements in performance and usability. Mol Biol Evol. 2013;30:772-80.

48. Kumar S, Nei M, Dudley J, Tamura K. MEGA: a biologist-centric software for evolutionary analysis of DNA and protein sequences. Briefs Bioinf. 2008;9: 299-306.

49. Felsenstein J. PHYLIP - Phylogeny inference package (version 3.2). Cladistics. 1989;5:164-6.

\section{Submit your next manuscript to BioMed Central and we will help you at every step:}

- We accept pre-submission inquiries

- Our selector tool helps you to find the most relevant journal

- We provide round the clock customer support

- Convenient online submission

- Thorough peer review

- Inclusion in PubMed and all major indexing services

- Maximum visibility for your research

Submit your manuscript at www.biomedcentral.com/submit 